Pacific Journal of Mathematics

POSITIVE ANALYTIC CAPACITY BUT ZERO BUFFON 


\section{POSITIVE ANALYTIC CAPACITY BUT ZERO BUFFON NEEDLE PROBABILITY}

Peter W. Jones and Takafumi Murai

There exists a compact set of positive analytic capacity but zero Buffon needle probability.

1. Introduction. For a compact set $E$ in the complex plane $\mathbf{C}$, $H^{\infty}\left(E^{c}\right)$ denotes the Banach space of bounded analytic functions outside $E$ with supremum norm $\|\cdot\|_{H^{\infty}\left(E^{c}\right)}$. The analytic capacity of $E$ is defined by

$$
\gamma(E)=\sup \left\{\left|f^{\prime}(\infty)\right| ; f \in H^{\infty}\left(E^{c}\right),\|f\|_{H^{\infty}\left(E^{c}\right)} \leq 1\right\},
$$

where $f^{\prime}(\infty)=\lim _{z \rightarrow \infty} z(f(z)-f(\infty))[1$, p. 6]. Let $\mathscr{L}(r, \theta)(r>$ $0,-\pi<\theta \leq \pi)$ denote the straight line defined by the equation $x \cos \theta+y \sin \theta=r$. The Buffon length of $E$ is defined by

$$
B u(E)=\iint_{\{(r, \theta) ; \mathscr{L}(r, \theta) \cap E \neq \varnothing\}} d r d \theta .
$$

Vitushkin [7] asked whether two classes of null-sets concerning $\gamma(\cdot)$ and $B u(\cdot)$ are same or not (cf. [2], [3]). Mattila [4] showed that these two classes are different. (He showed that the class of null-sets concerning $B u(\cdot)$ is not conformal invariant. Hence his method does not give the information about the implication between these two classes.) The second author [5] showed that, for any $0<\varepsilon<1$, there exists a compact set $E_{\varepsilon}$ such that $\gamma\left(E_{\varepsilon}\right)=1, B u\left(E_{\varepsilon}\right) \leq \varepsilon$. The purpose of this note is to show

Theorem. There exists a compact set $E_{0}$ such that $\gamma\left(E_{0}\right)=1$, $B u\left(E_{0}\right)=0$.

2. Cranks. To construct $E_{0}$, we begin by defining cranks. The 1dimension Lebesgue measure is denoted by $|\cdot|$. For a finite union $E$ of segments in $\mathbf{C}$, its length is also denoted by $|E|$. For $\rho>0, z \in \mathbf{C}$ and a set $E \subset \mathbf{C}$, we write $[\rho E+z]=\{\rho \zeta+z ; \zeta \in E\}$. With $0 \leq \varphi<1$ and a segment $J \subset C$ parallel to the $x$-axis, we associate the closed segment $J(\varphi)$ of the same midpoint as $J$, parallel to the $x$-axis and of 
length $(1+\varphi)|J|$. With a positive integer $q, 0 \leq \varphi<1$ and a segment $J$ parallel to the $x$-axis, we associate

$$
J(q, \varphi)=\bigcup_{k=1}^{2^{q-1}}\left[J_{2 k-1}(\varphi)+i 2^{-q}|J|\right] \cup \bigcup_{k=1}^{2^{q-1}} J_{2 k}(\varphi),
$$

where $\left\{J_{k}\right\}_{k=1}^{2^{q}}$ are mutually non-overlapping segments on $J$ of length $2^{-q}|J|$; they are ordered from left to right. The set $J(q, \varphi)$ is a union of $2^{q}$ closed segments of length $2^{-q}(1+\varphi)|J|$. The segment $\Gamma_{0}=$ $\{x ; 0 \leq x \leq 1\} \subset \mathbf{C}$ is called a crank of type 0 . For a finite sequence $\left\{\varphi_{j}\right\}_{j=0}^{n}, \varphi_{0}=0(n \geq 1)$ of non-negative numbers less than 1 , a finite union $\Gamma$ of closed segments is called a crank of type $\left\{\varphi_{j}\right\}_{j=0}^{n}$ if there exists a crank $\Gamma^{\prime}=\bigcup_{k=1}^{l} J_{k}\left(\left\{J_{k}\right\}_{k=1}^{l}\right.$ are components of $\left.\Gamma^{\prime}\right)$ of type $\left\{\varphi_{j}\right\}_{j=0}^{n-1}$ such that

$$
\Gamma=\bigcup_{k=1}^{l} J_{k}\left(q_{k}, \varphi_{n}\right)
$$

for some $l$-tuple $\left(q_{1}, \ldots, q_{l}\right)$ of positive integers larger than or equal to $q_{0}=100$. We write $\Gamma^{\prime}\left[\varphi_{n} \Gamma\right.$. For a sequence $\left\{\varphi_{j}\right\}_{j=0}^{\infty}, \varphi_{0}=0$ of non-negative numbers less than 1 , a set $\Gamma$ is called a crank of type $\left\{\varphi_{j}\right\}_{j=0}^{\infty}$, if there exists a sequence $\left\{\Gamma_{n}\right\}_{n=0}^{\infty}$ of cranks such that

$$
\Gamma_{n} \text { is of type }\left\{\varphi_{j}\right\}_{j=0}^{n} \text {, }
$$

$$
\begin{gathered}
\Gamma_{0}\left[\varphi _ { 1 } \Gamma _ { 1 } \left[\varphi_{2} \cdots,\right.\right. \\
\Gamma=\bigcap_{n=0}^{\infty} \overline{\bigcup_{j=n}^{\infty} \Gamma_{j} .}
\end{gathered}
$$

We write by $\mathbf{O}_{n}$ the finite sequence of $n$ zeros $(n \geq 1)$. For a finite union $\Gamma$ of segments, $L^{p}(\Gamma)(1 \leq p \leq \infty)$ denotes the $L^{p}$ space on $\Gamma$ with respect to the length element $|d \dot{z}|$. We define an operator $\mathscr{H}_{\Gamma}$ on $L^{p}(\Gamma)$ by

$$
\begin{aligned}
\mathscr{H}_{\Gamma} f(z) & =\frac{1}{2 \pi i} \text { p.v. } \int_{\Gamma} \frac{f(\zeta)}{\zeta-z}|d \zeta| \\
& =\frac{1}{2 \pi i} \lim _{\varepsilon \rightarrow 0} \int_{|\zeta-z|>\varepsilon, \zeta \in \Gamma} \frac{f(\zeta)}{\zeta-z}|d \zeta| .
\end{aligned}
$$

The following fact is already known. 
LEMMA 1 ([5]). For any positive integer $m$, there exist a crank $\Gamma_{m}^{*}$ of type $\mathbf{O}_{m+1}$ and a non-negative function $g_{m}^{*}$ on $\Gamma_{m}^{*}$ such that $g_{m}^{*}$ is a constant on each component of $\Gamma_{m}^{*}$,

$\left\|g_{m}^{*}\right\|_{L^{1}\left(\Gamma_{m}^{*}\right)}=1, \quad\left\|g_{m}^{*}\right\|_{L^{\infty}\left(\Gamma_{m}^{*}\right)} \leq C_{1}, \quad\left\|\operatorname{Re} \mathscr{K}_{\Gamma_{m}^{*}} g_{m}^{*}\right\|_{L^{\infty}\left(\Gamma_{m}^{*}\right)} \leq C_{1} \sqrt{m}$,

$$
B u\left(\Gamma_{m}^{*}\right) \leq C_{1} / m^{9 / 10},
$$

where $\operatorname{Re} \zeta$ is the real part of $\zeta$ and $C_{1}$ is an absolute constant.

Our method is as follows. We define a sequence $\{n(k)\}_{k=0}^{\infty}$ of nonnegative integers with large gaps. Choosing $\left\{\varphi_{j}\right\}_{j=0}^{10 n(1)}$ suitably, we define a crank $\Gamma_{10 n(1)}$ of type $\left\{\varphi_{j}\right\}_{j=0}^{10 n(1)}$. Then $\left|\Gamma_{10 n(1)}\right|=\prod_{\mu=1}^{10 n(1)}\left(1+\varphi_{\mu}\right)$. Replacing each component of $\Gamma_{10 n(1)}$ by a crank similar to $\Gamma_{n(2)-10 n(1)}^{*}$ in Lemma 1, we construct a crank $\Gamma_{n(2)}$ of type $\left\{\varphi_{j}\right\}_{j=0}^{n(2)}$, where $\varphi_{j}=0$ $(10 n(1)+1 \leq j \leq n(2))$. Then we see that

$$
\begin{gathered}
1 / \gamma\left(\Gamma_{n(2)}\right) \leq 1 / \gamma\left(\Gamma_{10 n(1)}\right)+\operatorname{Const}(n(2)-10 n(1))^{1 / 2} / \prod_{j=1}^{10 n(1)}\left(1+\varphi_{j}\right), \\
B u\left(\Gamma_{n(2)}\right) \leq C_{1} \prod_{j=1}^{10 n(1)}\left(1+\varphi_{j}\right)(n(2)-10 n(1))^{-9 / 10} .
\end{gathered}
$$

Our sequence $\left\{\varphi_{j}\right\}_{j=0}^{10 n(1)}$ is chosen so that

$$
n(2)-10 n(1)=\left\{\prod_{j=1}^{10 n(1)}\left(1+\varphi_{j}\right)\right\}^{4 / 3}
$$

Hence

$$
\begin{gathered}
1 / \gamma\left(\Gamma_{n(2)}\right) \leq 1 / \gamma\left(\Gamma_{10 n(1)}\right)+\operatorname{Const}(n(2)-10 n(1))^{-1 / 4}, \\
B u\left(\Gamma_{n(2)}\right) \leq C_{1}(n(2)-10 n(1))^{-3 / 20} .
\end{gathered}
$$

Replacing each component of $\Gamma_{n(2)}$ by a suitable crank, we construct a crank $\Gamma_{10 n(2)}$ of type $\left\{\varphi_{j}\right\}_{j=0}^{10 n(2)}$. Replacing each component of $\Gamma_{10 n(2)}$ by a crank similar to $\Gamma_{n(3)-10 n(2)}^{*}$, we construct a crank $\Gamma_{n(3)}$ of type $\left\{\varphi_{j}\right\}_{j=0}^{n(3)}$, where $\varphi_{j}=0(10 n(2)+1 \leq j \leq n(3))$. The sequence $\left\{\varphi_{j}\right\}_{j=n(2)+1}^{10 n(2)}$ is chosen so that $\left|(n(3)-10 n(2))-\left(\prod_{j=1}^{10 n(2)}\left(1+\varphi_{j}\right)\right)^{4 / 3}\right|$ is small. We see that

$$
\begin{gathered}
1 / \gamma\left(\Gamma_{n(3)}\right) \leq 1 / \gamma\left(\Gamma_{10 n(1)}\right)+\operatorname{Const}(n(2)-10 n(1))^{-1 / 4} \\
+\operatorname{Const}(n(3)-10 n(2))^{-1 / 4}+(\text { negligible quantity }), \\
B u\left(\Gamma_{n(3)}\right) \leq C_{1}(n(3)-10 n(2))^{-3 / 20}
\end{gathered}
$$


Repeating this argument, we define a sequence $\left\{\Gamma_{n(k)}\right\}_{k=2}^{\infty}$ of cranks such that

$$
\limsup _{k \rightarrow \infty} 1 / \gamma\left(\Gamma_{n(k)}\right)<\infty, \quad \lim _{k \rightarrow \infty} B u\left(\Gamma_{n(k)}\right)=0 .
$$

Then the analytic capacity of the limit crank is positive and its Buffon length is zero.

\section{Lemmas.}

LEMMA 2. Let $\Gamma_{n}$ be a crank of type $\left\{\varphi_{j}\right\}_{j=0}^{n}, g_{n}$ be a non-negative function on $\Gamma_{n}$ such that $g_{n}$ is a constant on each component of $\Gamma_{n}$, and let $\left\{\varphi_{j}\right\}_{j=n+1}^{n+m}$ be non-negative numbers less than 1. Then there exist a crank $\Gamma_{n+m}$ of type $\left\{\varphi_{j}\right\}_{j=0}^{n+m}$ and a non-negative function $g_{n+m}$ on $\Gamma_{n+m}$ such that

$$
g_{n+m} \text { is a constant on each component of } \Gamma_{n+m},
$$

$$
\left\|g_{n+m}\right\|_{L^{1}\left(\Gamma_{n+m}\right)}=\left\|g_{n}\right\|_{L^{1}\left(\Gamma_{n}\right)}
$$

$$
\left\|g_{n+m}\right\|_{L^{\infty}\left(\Gamma_{n+m}\right)} \leq\left\|g_{n}\right\|_{L^{\infty}\left(\Gamma_{n}\right)} / \prod_{\mu=n+1}^{n+m}\left(1+\varphi_{\mu}\right)
$$

(7) $\left\|\operatorname{Re} \mathscr{H}_{\Gamma_{n+m}} g_{n+m}\right\|_{L^{\infty}\left(\Gamma_{n+m}\right)}$

$$
\leq\left\|\operatorname{Re} \mathscr{H}_{\Gamma_{n}} g_{n}\right\|_{L^{\infty}\left(\Gamma_{n}\right)}+\left\|g_{n}\right\|_{L^{\infty}\left(\Gamma_{n}\right)} \sum_{j=n+1}^{n+m}\left\{1 / \prod_{\mu=n+1}^{j}\left(1+\varphi_{\mu}\right)\right\} .
$$
put

We can write $\Gamma_{n}=\bigcup_{k=1}^{l_{n}} J_{k}^{(n)}$ with its components $\left\{J_{k}^{(n)}\right\}_{k=1}^{l_{n}}$. We

$$
\Gamma_{n+1}=\bigcup_{k=1}^{l_{n}} J_{k}^{(n)}\left(q_{n+1}, \varphi_{n+1}\right)
$$

where $q_{n+1}\left(\geq q_{0}=100\right)$ is determined later. Suppose that $\left\{\Gamma_{\mu}\right\}_{\mu=n+1}^{j}$ have been defined. We can write $\Gamma_{j}=\bigcup_{k=1}^{l_{j}} J_{k}^{(j)}$ with its components $\left\{J_{k}^{(j)}\right\}_{k=1}^{l_{j}}$. We put

$$
\Gamma_{j+1}=\bigcup_{k=1}^{l_{j}} J_{k}^{(j)}\left(q_{j+1}, \varphi_{j+1}\right)
$$


Thus $\left\{\Gamma_{j}\right\}_{j=n+1}^{n+m}$ are defined; $\left\{q_{j}\right\}_{j=n+1}^{n+m}$ are determined later. Let $n+$ $1 \leq j \leq n+m$. We define a non-negative function $g_{j}$ on $\Gamma_{j}$ as follows. Each component $J_{k}^{(n)}$ of $\Gamma_{n}$ generates $2^{q_{n+1}+\cdots+q_{j}}$ components of $\Gamma_{j}$. On these components, we put

$$
g_{j}(z)=\left\{\frac{1}{\left|J_{k}^{(n)}\right|} \int_{J_{k}^{(n)}} g_{n}(\zeta)|d \zeta|\right\} / \prod_{\mu=n+1}^{j}\left(1+\varphi_{\mu}\right)
$$

Since the total length of these $2^{q_{n+1}+\cdots+q}$ components is

$$
\left|J_{k}^{(n)}\right| \prod_{\mu=n+1}^{j}\left(1+\varphi_{\mu}\right)
$$

the integration of $g_{j}$ over these components is equal to $\int_{J_{k}^{(n)}} g_{n}(\zeta)|d \zeta|$. Hence $\left\|g_{j}\right\|_{L^{\prime}\left(\Gamma_{j}\right)}=\left\|g_{n}\right\|_{L^{\prime}\left(\Gamma_{n}\right)}$. Evidently, $g_{j}$ is a constant on each component of $\Gamma_{j}$. We have

$$
\left\|g_{j}\right\|_{L^{\infty}\left(\Gamma_{j}\right)} \leq\left\|g_{n}\right\|_{L^{\infty}\left(\Gamma_{n}\right)} / \prod_{\mu=n+1}^{j}\left(1+\varphi_{\mu}\right) .
$$

In particular, (4)-(6) hold. To prove (7), we estimate

$$
\left\|\operatorname{Re} \mathscr{F}_{\Gamma_{j+1}} g_{j+1}\right\|_{L^{\infty}\left(\Gamma_{j+1}\right)} .
$$

Recall (8). We have

$$
\begin{aligned}
J_{k}^{(j)}\left(q_{j+1}, \varphi_{j+1}\right)= & \bigcup_{\mu=1}^{\sigma_{j+1}}\left[J_{k, 2 \mu}^{(j)}\left(\varphi_{j+1}\right)+i 2^{-q_{j+1}}\left|J_{k}^{(j)}\right|\right] \\
& \cup \bigcup_{\mu=1}^{\sigma_{j+1}} J_{k, 2 \mu-1}^{(j)}\left(\varphi_{j+1}\right) \quad\left(\sigma_{j+1}=2^{q_{j+1}-1}, 1 \leq k \leq l_{j}\right),
\end{aligned}
$$

where $\left\{J_{k, \mu}^{(j)}\right\}_{\mu=1}^{2 \sigma_{j+1}}$ are mutually non-overlapping segments on $J_{k}^{(j)}$ of 
length $2^{-q_{\jmath+1}}\left|J_{k}^{(j)}\right|$; they are ordered from left to right. Let

$$
z_{0} \in \bigcup_{\mu=1}^{\sigma_{j+1}}\left[J_{k_{0}, 2 \mu}^{(j)}\left(\varphi_{j+1}\right)+i 2^{-q_{j+1}}\left|J_{k_{0}}^{(j)}\right|\right]
$$

and let $z_{0}^{*}$ be the nearest point on $J_{k_{0}}^{(j)}$ to $z_{0}$. Then

$$
\begin{aligned}
L_{1}=\left|\operatorname{Re} \frac{1}{2 \pi i} \mathrm{p} \cdot \mathrm{v} \cdot \int_{J_{k_{0}}^{(J)}\left(q_{j+1}, \varphi_{j+1}\right)} \frac{g_{j+1}(\zeta)}{\zeta-z_{0}}\right| d \zeta \mid \\
\quad-\operatorname{Re} \frac{1}{2 \pi i} \mathrm{p} \cdot \mathrm{v} \cdot \int_{J_{k_{0}}^{(J)}} \frac{g_{j}(\zeta)}{\zeta-z_{0}^{*}}|d \zeta| \mid \\
=\left|\operatorname{Re} \frac{1}{2 \pi i} \sum_{\mu=1}^{\sigma_{j+1}} \mathrm{p} \cdot \mathrm{v} \cdot \int_{J_{k_{0} \cdot 2 \mu-1}^{(J)}\left(\varphi_{j+1}\right)} \frac{g_{j+1}(\zeta)}{\zeta-z_{0}}\right| d \zeta|| \\
\leq \frac{1}{2 \pi} \int_{-\infty}^{\infty} \frac{2^{-q_{j+1} \mid}\left|J_{k_{0}}^{(j)}\right|}{\left(x-\operatorname{Re} z_{0}\right)^{2}+\left(2^{-q_{j+1}}\left|J_{k_{0}}^{(j)}\right|\right)^{2}}\left\|g_{j+1}\right\|_{L^{\infty}\left(\Gamma_{J+1}\right)} d x \\
\left.\leq\left\|g_{n}\right\|_{L^{\infty}\left(\Gamma_{n}\right) /} / \prod_{\mu=n+1}^{j+1}\left(1+\varphi_{\mu}\right)\right\} .
\end{aligned}
$$

Let

$$
\rho_{j}=\min _{1 \leq k \leq l_{j}} \operatorname{dis}\left(J_{k}^{(j)}, \Gamma_{j}-J_{k}^{(j)}\right), \quad \tau\left(q_{j+1}\right)=2^{-q_{j+1}} \max _{1 \leq k \leq l_{j}}\left|J_{k}^{(j)}\right|
$$

where $\operatorname{dis}(\cdot, \cdot)$ is the distance. We choose, for a while, $q_{j+1}\left(\geq q_{0}\right)$ so that $\tau\left(q_{j+1}\right) \leq \rho_{j} / 10$. Since

$$
\begin{gathered}
\int_{\left[J_{k, 2 \mu}^{(J)}\left(\varphi_{j+1}\right)+i 2^{\left.-q_{j+1}\left|J_{k}^{(J)}\right|\right]}\right.} g_{j+1}(\zeta)|d \zeta|=\int_{J_{k, 2 \mu}^{(J)}} g_{j}(\zeta)|d \zeta|, \\
\int_{J_{k, 2 \mu-1}^{(J)}\left(\varphi_{j+1}\right)} g_{j+1}(\zeta)|d \zeta|=\int_{J_{k, 2 \mu-1}^{(j)}} g_{j}(\zeta)|d \zeta| \\
\left(1 \leq k \leq l_{j}, 1 \leq \mu \leq 2^{q_{J+1}-1}\left(=\sigma_{j+1}\right)\right),
\end{gathered}
$$


we have

$$
\begin{aligned}
& L_{2}=\left|\operatorname{Re} \frac{1}{2 \pi i} \int_{\Gamma_{\jmath+1}-J_{k_{0}}^{(J)}\left(q_{j+1}, \varphi_{j+1}\right)} \frac{g_{j+1}(\zeta)}{\zeta-z_{0}}\right| d \zeta \mid \\
& -\operatorname{Re} \frac{1}{2 \pi i} \int_{\Gamma_{j}-J_{k_{0}}^{(\jmath)}} \frac{g_{j}(\zeta)}{\zeta-z_{0}^{*}}|d \zeta| \\
& \leq \frac{1}{2 \pi} \sum_{k \neq k_{0}}\left\{\sum_{\mu=1}^{\sigma_{j+1}}\left|\int_{\left[J_{k, 2 \mu}^{(j)}\left(\varphi_{j+1}\right)+i 2^{\left.-q_{j+1}\left|J_{k}^{(j)}\right|\right]}\right.} \frac{g_{j+1}(\zeta)}{\zeta-z_{0}}\right| d \zeta \mid\right. \\
& -\int_{J_{k, 2 \mu}^{(J)}} \frac{g_{j}(\zeta)}{\zeta-z_{0}^{*}}|d \zeta| \\
& \left.+\sum_{\mu=1}^{\sigma_{J+1}}\left|\int_{J_{k, 2 \mu-1}^{(J)}\left(\varphi_{j+1}\right)} \frac{g_{j+1}(\zeta)}{\zeta-z_{0}}\right| d \zeta\left|-\int_{J_{k, 2 \mu-1}^{(j)}} \frac{g_{j}(\zeta)}{\zeta-z_{0}^{*}}\right| d \zeta||\right\} \\
& \leq \text { Const } \tau\left(q_{j+1}\right) \rho_{j}^{-2} \sum_{k \neq k_{0}} \sum_{\mu=1}^{2^{q_{j+1}}} \int_{J_{k, \mu}^{(j)}} g_{j}(\zeta)|d \zeta| \\
& \leq \text { Const } \tau\left(q_{j+1}\right) \rho_{j}^{-2}\left\|g_{j}\right\|_{L^{1}\left(\Gamma_{j}\right)} \\
& =\text { Const } \tau\left(q_{j+1}\right) \rho_{j}^{-2}\left\|g_{n}\right\|_{L^{1}\left(\Gamma_{n}\right)} \text {. }
\end{aligned}
$$

\section{Thus}

(9) $\left|\operatorname{Re} \mathscr{K}_{\Gamma_{j+1}} g_{j+1}\left(z_{0}\right)\right| \leq\left|\operatorname{Re} \mathscr{K}_{\Gamma_{j}} g_{j}\left(z_{0}^{*}\right)\right|+L_{1}+L_{2}$

$$
\begin{aligned}
\leq & \left\|\operatorname{Re} \mathscr{H}_{\Gamma_{j}} g_{j}\right\|_{L^{\infty}\left(\Gamma_{j}\right)}+\left\|g_{n}\right\|_{L^{\infty}\left(\Gamma_{n}\right)} /\left\{2 \prod_{\mu=n+1}^{j+1}\left(1+\varphi_{\mu}\right)\right\} \\
& +\operatorname{Const} \tau\left(q_{j+1}\right) \rho_{j}^{-2}\left\|g_{n}\right\|_{L^{1}\left(\Gamma_{n}\right)} .
\end{aligned}
$$

In the same manner, we have (9) for any point $z_{0}$ in

$$
\bigcup_{\mu=1}^{\sigma_{j+1}} J_{k_{0}, 2 \mu-1}^{(j)}\left(\varphi_{j+1}\right)
$$


Since $k_{0}\left(1 \leq k_{0} \leq l_{j}\right)$ is arbitrary, $\left\|\operatorname{Re} \mathscr{H}_{\Gamma_{j+1}} g_{j+1}\right\|_{L^{\infty}\left(\Gamma_{\jmath+1}\right)}$ is dominated by the summation of the last three quantities in (9). Consequently,

(10) $\left\|\operatorname{Re} \mathscr{H}_{\Gamma_{n+m}} g_{n+m}\right\|_{L^{\infty}\left(\Gamma_{n+m}\right)}$

$$
\begin{aligned}
& \leq\left\|\operatorname{Re} \mathscr{H}_{\Gamma_{n+m-1}} g_{n+m-1}\right\|_{L^{\infty}\left(\Gamma_{n+m-1}\right)} \\
& +\left\|g_{n}\right\|_{L^{\infty}\left(\Gamma_{n}\right)} /\left\{2 \prod_{\mu=n+1}^{n+m}\left(1+\varphi_{\mu}\right)\right\} \\
& + \text { Const } \tau\left(q_{n+m}\right) \rho_{n+m-1}^{-2}\left\|g_{n}\right\|_{L^{1}\left(\Gamma_{n}\right)} \leq \cdots \leq\left\|\operatorname{Re} \mathscr{H}_{\Gamma_{n}} g_{n}\right\|_{L^{\infty}\left(\Gamma_{n}\right)} \\
& +\left\|g_{n}\right\|_{L^{\infty}\left(\Gamma_{n}\right)} \sum_{j=n+1}^{n+m} 1 /\left\{2 \prod_{\mu=n+1}^{j}\left(1+\varphi_{\mu}\right)\right\} \\
& + \text { Const }\left\|g_{n}\right\|_{L^{1}\left(\Gamma_{n}\right)} \sum_{j=n+1}^{n+m} \tau\left(q_{j}\right) \rho_{j-1}^{-2} .
\end{aligned}
$$

Since $\lim _{q \rightarrow \infty} \tau(q)=0$, we can inductively define $\left\{q_{j}\right\}_{j=n+1}^{n+m}$ so that (7) holds. This completes the proof of Lemma 2.

LEMMA 3. Let $\Gamma_{n}$ be a crank of type $\left\{\varphi_{j}\right\}_{j=0}^{n}, g_{n}$ be a non-negative function on $\Gamma_{n}$ such that $g_{n}$ is a constant on each component of $\Gamma_{n}$, and let $m$ be a positive integer. Then there exist a crank $\Gamma_{n+m}$ of type $\left\{\varphi_{j}\right\}_{j=0}^{n+m}$ with $\varphi_{j}=0(n+1 \leq j \leq n+m)$ and a non-negative function $g_{n+m}$ on $\Gamma_{n+m}$ such that

$$
g_{n+m} \text { is a constant on each component of } \Gamma_{n+m} \text {, }
$$

$$
\begin{gathered}
\left\|g_{n+m}\right\|_{L^{1}\left(\Gamma_{n+m}\right)}=\left\|g_{n}\right\|_{L^{1}\left(\Gamma_{n}\right)}, \\
\left\|g_{n+m}\right\|_{L^{\infty}\left(\Gamma_{n+m}\right)} \leq C_{1}\left\|g_{n}\right\|_{L^{\infty}\left(\Gamma_{n}\right)}, \\
\left\|\operatorname{Re} \mathscr{K}_{\Gamma_{n+m}} g_{n+m}\right\|_{L^{\infty}\left(\Gamma_{n+m}\right)} \leq \mathscr{G}_{\Gamma_{n}} g_{n}\left\|_{L^{\infty}\left(\Gamma_{n}\right)}+C_{2} \sqrt{m}\right\| g_{n} \|_{L^{\infty}\left(\Gamma_{n}\right)}, \\
\leq \| \operatorname{Re}\left(\Gamma_{n+m}\right) \leq C_{1}\left|\Gamma_{n}\right| / m^{9 / 10},
\end{gathered}
$$

where $C_{1}$ is the constant in Lemma 1 and $C_{2}$ is an absolute constant.

We can write $\Gamma_{n}=\bigcup_{k=1}^{l} J_{k}$ with its components $\left\{J_{k}\right\}_{k=1}^{l}$. Let $z_{k}$ be the left endpoint of $J_{k}(1 \leq k \leq l)$. We put

$$
\Gamma_{n+m}=\bigcup_{k=1}^{l} \Lambda_{k}, \quad \Lambda_{k}=\left[\left|J_{k}\right| \Gamma_{m}^{*}+z_{k}\right]
$$




$$
g_{n+m}(z)=g_{m}^{*}\left(\left(z-z_{k}\right) /\left|J_{k}\right|\right) g_{n}\left(z_{k}\right) \quad\left(z \in \Lambda_{k}, 1 \leq k \leq l\right),
$$

where $\Gamma_{m}^{*}, g_{m}^{*}$ are the crank and the function in Lemma 1, respectively. Then $\Gamma_{n+m}$ is a crank of type $\left\{\varphi_{j}\right\}_{j=0}^{n+m}$. Evidently, (11) and (12) hold. Lemma 1 immediately yields (13) and (15). Let $z_{0} \in \Lambda_{k_{0}}$ and let $z_{0}^{*}$ be the projection of $z_{0}$ to $J_{k_{0}}$. Then Lemma 1 shows that

$$
\begin{aligned}
\left|\operatorname{Re} \mathscr{H}_{\Gamma_{n+m}} g_{n+m}\left(z_{0}\right)-\operatorname{Re} \mathscr{H}_{\Gamma_{n}} g_{n}\left(z_{0}^{*}\right)\right| \\
\quad \leq\left|\operatorname{Re} \frac{1}{2 \pi i} \int_{\Lambda_{k_{0}}} \frac{g_{n+m}(\zeta)}{\zeta-z_{0}}\right| d \zeta\left|-\operatorname{Re} \frac{1}{2 \pi i} \int_{J_{k_{0}}} \frac{g_{n}(\zeta)}{\zeta-z_{0}^{*}}\right| d \zeta||+\frac{1}{2 \pi} L^{0} \\
\quad=\left|\operatorname{Re} \frac{1}{2 \pi i} \int_{\Lambda_{k_{0}}} \frac{g_{n+m}(\zeta)}{\zeta-z_{0}}\right| d \zeta||+\frac{1}{2 \pi} L^{0} \\
\quad=\left|\operatorname{Re}\left(\mathscr{H}_{\Gamma_{m}^{*}} g_{m}^{*}\right)\left(\frac{z_{0}-z_{k}}{\left|J_{k}\right|}\right)\right| g_{n}\left(z_{k}\right)+\frac{1}{2 \pi} L^{0} \\
\quad \leq C_{1} \sqrt{m}\left\|g_{n}\right\|_{L^{\infty}\left(\Gamma_{n}\right)}+\frac{1}{2 \pi} L^{0},
\end{aligned}
$$

where

$$
L^{0}=\sum_{k \neq k_{0}}\left|\int_{\Lambda_{k}} \frac{g_{n+m}(\zeta)}{\zeta-z_{0}}\right| d \zeta\left|-\int_{J_{k}} \frac{g_{n}(\zeta)}{\zeta-z_{0}^{*}}\right| d \zeta|| .
$$

Let $\left\{\Gamma_{j}\right\}_{j=0}^{n}$ be cranks such that

$$
\Gamma_{0}\left[\varphi _ { 1 } \Gamma _ { 1 } \left[\varphi _ { 2 } \cdots \left[\varphi_{n} \Gamma_{n} .\right.\right.\right.
$$

For $1 \leq k \leq l, 0 \leq j \leq n, \gamma_{k}(j)$ denotes the component of $\Gamma_{j}$ generating $J_{k}$. In particular, $\gamma_{k}(n)=J_{k}(1 \leq k \leq l)$. We put

$$
L_{j}^{0}=\sum_{k \in \mathscr{F}_{J}}\left|\int_{\Lambda_{k}} \frac{g_{n+m}(\zeta)}{\zeta-z_{0}}\right| d \zeta\left|-\int_{J_{k}} \frac{g_{n}(\zeta)}{\zeta-z_{0}^{*}}\right| d \zeta|| \quad(1 \leq j \leq n),
$$

where

$$
\mathscr{F}_{j}=\left\{1 \leq k \leq l ; k \neq k_{0}, \gamma_{k}(j-1)=\gamma_{k_{0}}(j-1), \gamma_{k}(j) \neq \gamma_{k_{0}}(j)\right\} .
$$

Then

$$
L^{0}=\sum_{j=1}^{n} L_{j}^{0}
$$

Since $\Gamma_{m}^{*}$ is a crank of type $\mathbf{O}_{m+1}$, a geometric observation shows that, for any $z \in \Lambda_{k}(1 \leq k \leq l)$,

$$
\operatorname{dis}\left(z, J_{k}\right) \leq 2\left|J_{k}\right|\left\{2^{-q_{0}}+2^{-2 q_{0}}+\cdots+2^{-m q_{0}}\right\} \leq \frac{1}{100}\left|J_{k}\right|
$$


Hence $\Lambda_{k}$ is contained in the square $Q_{k}=\left\{z+i s ; z \in J_{k}, 0 \leq s \leq\right.$ $\left.\left|J_{k}\right| / 100\right\}(1 \leq k \leq l)$. Since $\left|\gamma_{k}(n)\right|=\left|\gamma_{k_{0}}(n)\right|\left(k \in \mathscr{F}_{n}\right)$, we have, for $k \in \mathscr{F}_{n}$,

$$
\begin{aligned}
\operatorname{dis}\left(Q_{k}, Q_{k_{0}}\right) & \geq \operatorname{dis}\left(\gamma_{k}(n), \gamma_{k_{0}}(n)\right)-\frac{1}{100}\left\{\left|\gamma_{k}(n)\right|+\left|\gamma_{k_{0}}(n)\right|\right\} \\
& =\operatorname{dis}\left(\gamma_{k}(n), \gamma_{k_{0}}(n)\right)-\frac{1}{50}\left|\gamma_{k_{0}}(n)\right| .
\end{aligned}
$$

For any $1 \leq j \leq n-1, z \in Q_{k}$,

$$
\begin{aligned}
& \operatorname{dis}\left(z, \gamma_{k}(j)\right) \leq \sum_{\mu=j+1}^{n}\left\{\frac{\left|\gamma_{k}(\mu)\right|}{\left(1+\varphi_{\mu}\right)}+\left|\gamma_{k}(\mu)\right|\right\}+\frac{1}{100}\left|J_{k}\right| \\
& \leq 2\left|\gamma_{k}(j)\right| \sum_{\mu=j+1}^{n}\left|\gamma_{k}(\mu)\right| /\left|\gamma_{k}(j)\right|+\frac{1}{100}\left|\gamma_{k}(j)\right| \\
& \leq 2\left|\gamma_{k}(j)\right|\left\{2^{-q_{0}}\left(1+\varphi_{j+1}\right)+2^{-2 q_{0}}\left(1+\varphi_{j+1}\right)\left(1+\varphi_{j+2}\right)\right. \\
& \left.\quad+\cdots+2^{-(n-j) q_{0}}\left(1+\varphi_{j+1}\right) \cdots\left(1+\varphi_{n}\right)\right\}+\frac{1}{100}\left|\gamma_{k}(j)\right| \\
& \leq 2\left|\gamma_{k}(j)\right|\left\{2^{-\left(q_{0}-1\right)}+2^{-2\left(q_{0}-1\right)}+\cdots\right\}+\frac{1}{100}\left|\gamma_{k}(j)\right| \leq \frac{1}{50}\left|\gamma_{k}(j)\right| .
\end{aligned}
$$

Since $\left|\gamma_{k}(j)\right|=\left|\gamma_{k_{0}}(j)\right|\left(k \in \mathscr{F}_{j}\right)$, we have, for $k \in \mathscr{F}_{j}, 1 \leq j \leq n-1$,

$$
\begin{aligned}
\operatorname{dis}\left(Q_{k}, Q_{k_{0}}\right) & \geq \operatorname{dis}\left(\gamma_{k}(j), \gamma_{k_{0}}(j)\right)-\frac{1}{50}\left\{\left|\gamma_{k}(j)\right|+\left|\gamma_{k_{0}}(j)\right|\right\} \\
& =\operatorname{dis}\left(\gamma_{k}(j), \gamma_{k_{0}}(j)\right)-\frac{1}{25}\left|\gamma_{k_{0}}(j)\right| .
\end{aligned}
$$

Thus (16) holds for any $k \in \mathscr{F}_{j}, 1 \leq j \leq n$. Let $1 \leq j \leq n$. Since

$$
\int_{\Lambda_{k}} g_{n+m}(\zeta)|d \zeta|=\int_{J_{k}} g_{n}(\zeta)|d \zeta| \quad(1 \leq k \leq l)
$$

we have

$$
\begin{aligned}
& L_{j}^{0}= \sum_{k \in \mathscr{F}_{j}}\left|\int_{\Lambda_{k}}\left\{\frac{1}{\zeta-z_{0}}-\frac{1}{z_{k}-z_{0}^{*}}\right\} g_{n+m}(\zeta)\right| d \zeta \mid \\
& \quad+\int_{J_{k}}\left\{\frac{1}{z_{k}-z_{0}^{*}}-\frac{1}{\zeta-z_{0}^{*}}\right\} g_{n}(\zeta)|d \zeta| \mid \\
& \leq \text { Const } \sum_{k \in \mathscr{F}_{J}}\left(\left|J_{k}\right|+\left|J_{k_{0}}\right|\right) \operatorname{dis}\left(Q_{k}, Q_{k_{0}}\right)^{-2} \int_{J_{k}} g_{n}(\zeta)|d \zeta| \\
& \leq \text { Const }\left\|g_{n}\right\|_{L^{\infty}\left(\Gamma_{n}\right)} \sum_{k \in \mathscr{F}_{j}}\left(\left|J_{k}\right|+\left|J_{k_{0}}\right|\right)\left|J_{k}\right| \operatorname{dis}\left(Q_{k}, Q_{k_{0}}\right)^{-2} .
\end{aligned}
$$


The segment $\gamma_{k_{0}}(j-1)$ generates $2^{q_{j}}$ components $\left\{\lambda_{\nu}\right\}_{\nu=1}^{q_{j}}$ of $\Gamma_{j}$ of length $\left|\gamma_{k_{0}}(j)\right|$, where $q_{j}=\log \left\{\left(1+\varphi_{j}\right)\left|\gamma_{k_{0}}(j-1)\right| /\left|\gamma_{k_{0}}(j)\right|\right\} / \log 2\left(\geq q_{0}\right)$. We may assume that $\lambda_{1}=\gamma_{k_{0}}(j)$. Let

$$
\mathscr{F}_{j, \nu}=\left\{k \in \mathscr{F}_{j} ; \lambda_{\nu}=\gamma_{k}(j)\right\} \quad\left(2 \leq \nu \leq 2^{q_{j}}\right) .
$$

Then $\mathscr{F}_{j}=\bigcup_{\nu=2}^{2^{q_{j}}} \mathscr{F}_{j, \nu}$. We have, for $2 \leq \nu \leq 2^{q_{j}}$,

$$
\begin{aligned}
& \sum_{k \in \mathscr{F}_{j, \nu}}\left(\left|J_{k}\right|+\left|J_{k_{0}}\right|\right)\left|J_{k}\right| \\
& \quad \leq\left|\lambda_{1}\right| 2^{-q_{0}(n-j)} \prod_{j<\mu \leq n}\left(1+\varphi_{\mu}\right) \sum_{k \in \mathscr{F}_{j, \nu}}\left|J_{k}\right| \\
& \quad=\left|\lambda_{1}\right|^{2} 2^{-q_{0}(n-j)}\left\{\prod_{j<\mu \leq n}\left(1+\varphi_{\mu}\right)\right\}^{2} \leq\left|\lambda_{1}\right|^{2} 2^{-\left(q_{0}-2\right)(n-j),}
\end{aligned}
$$

where $\prod_{j<\mu \leq n}\left(1+\varphi_{\mu}\right)$ denotes 1 if $j=n$.

Hence a geometric observation and (16) show that the last quantity in (17) is dominated by

$$
\begin{aligned}
& \text { Const }\left\|g_{n}\right\|_{L^{\infty}\left(\Gamma_{n}\right)} \sum_{\nu=2}^{2^{q_{j}}} \sum_{k \in \mathscr{F}_{j, \nu}}\left(\left|J_{k}\right|+\left|J_{k_{0}}\right|\right)\left|J_{k}\right| \operatorname{dis}\left(Q_{k}, Q_{k_{0}}\right)^{-2} \\
& \leq \mathrm{Const}\left\|g_{n}\right\|_{L^{\infty}\left(\Gamma_{n}\right)} \sum_{\nu=2}^{2^{q_{j}}} \operatorname{dis}\left(\lambda_{\nu}, \lambda_{1}\right)^{-2} \sum_{k \in \mathscr{F}_{j, \nu}}\left(\left|J_{k}\right|+\left|J_{k_{0}}\right|\right)\left|J_{k}\right| \\
& \leq \mathrm{Const}\left\|g_{n}\right\|_{L^{\infty}\left(\Gamma_{n}\right)}\left|\lambda_{1}\right|^{2} 2^{-\left(q_{0}-2\right)(n-j)} \sum_{\nu=2}^{2^{q_{j}}} \operatorname{dis}\left(\lambda_{\nu}, \lambda_{1}\right)^{-2} \\
& \leq \mathrm{Const}\left\|g_{n}\right\|_{L^{\infty}\left(\Gamma_{n}\right)}\left|\lambda_{1}\right|^{2} 2^{-\left(q_{0}-2\right)(n-j)} \sum_{\mu=1}^{\infty}\left(\left|\lambda_{1}\right| \mu\right)^{-2} \\
& \leq \text { Const }\left\|g_{n}\right\|_{L^{\infty}\left(\Gamma_{n}\right)} 2^{-\left(q_{0}-2\right)(n-j)} .
\end{aligned}
$$

Thus

$$
\begin{aligned}
\left|\operatorname{Re} \mathscr{K}_{\Gamma_{n+m}} g_{n+m}\left(z_{0}\right)\right| \leq & \left|\operatorname{Re} \mathscr{H}_{\Gamma_{n}} g_{n}\left(z_{0}^{*}\right)\right| \\
& +C_{1} \sqrt{m}\left\|g_{n}\right\|_{L^{\infty}\left(\Gamma_{n}\right)}+\frac{1}{2 \pi} \sum_{j=1}^{n} L_{j}^{0} \\
\leq & \left\|\operatorname{Re} \mathscr{K}_{\Gamma_{n}} g_{n}\right\|_{L^{\infty}\left(\Gamma_{n}\right)}+C_{1} \sqrt{m}\left\|g_{n}\right\|_{L^{\infty}\left(\Gamma_{n}\right)} \\
& + \text { Const }\left\|g_{n}\right\|_{L^{\infty}\left(\Gamma_{n}\right)} \sum_{j=1}^{n} 2^{-\left(q_{0}-2\right)(n-j)},
\end{aligned}
$$


which shows that

$$
\left|\operatorname{Re} \mathscr{H}_{\Gamma_{n+m}} g_{n+m}\left(z_{0}\right)\right| \leq\left\|\operatorname{Re} \mathscr{H}_{\Gamma_{n}} g_{n}\right\|_{L^{\infty}\left(\Gamma_{n}\right)}+C_{2} \sqrt{m}\left\|g_{n}\right\|_{L^{\infty}\left(\Gamma_{n}\right)}
$$

for some absolute constant $C_{2}$. Since $z_{0} \in \Gamma_{n+m}$ is arbitrary, this gives (14). This completes the proof of Lemma 3.

LEMMA 4. Let $\Gamma$ be a crank of type $\left\{\varphi_{j}\right\}_{j=0}^{\infty}$, and let $\left\{\Gamma_{n}\right\}_{n=0}^{\infty}$ be a sequence of cranks satisfying (1)-(3). If $\liminf _{n \rightarrow \infty} B u\left(\Gamma_{n}\right)=0$, then $B u(\Gamma)=0$.

Let $\mathscr{P}^{\theta}(-\pi / 2<\theta \leq \pi / 2)$ denote the straight line defined by the equation $x \sin \theta-y \cos \theta=0$. For a set $E \subset \mathbf{C}, \operatorname{proj}_{\theta}(E)$ denotes the projection of $E$ to $\mathscr{P} \theta$. We have

$$
B u(E)=\int_{-\pi / 2}^{\pi / 2}\left|\operatorname{proj}_{\theta}(E)\right| d \theta .
$$

We can write $\Gamma_{n}=\bigcup_{k=1}^{l_{n}} J_{k}^{(n)}$ with its components $\left\{J_{k}^{(n)}\right\}_{k=1}^{l_{n}}$. In the same manner as in the proof of (14), we have

$$
\Gamma \subset \bigcup_{k=1}^{l_{n}}\left\{z ; \operatorname{dis}\left(z, J_{k}^{(n)}\right) \leq\left|J_{k}^{(n)}\right|\right\} \quad\left(=\bigcup_{k=1}^{l_{n}} R_{k}^{(n)} \text {, say }\right) \text {. }
$$

Hence, for any $-\pi / 2<\theta \leq \pi / 2$,

$$
\left|\operatorname{proj}_{\theta}(\Gamma)\right| \leq\left|\operatorname{proj}_{\theta}\left(\bigcup_{k=1}^{l_{n}} R_{k}^{(n)}\right)\right|
$$

We can decompose $\left\{k ; 1 \leq k \leq l_{n}\right\}$ into a finite number of mutually disjoint sets $\left\{\mathscr{G}_{\mu}^{\theta}\right\}_{\mu=1}^{\nu_{\theta}}$ so that $\operatorname{proj}_{\theta}\left(\bigcup_{k \in \mathscr{S}_{\mu}{ }^{\theta}} J_{k}^{(n)}\right)$ is connected. Then a geometric observation shows that

$$
\begin{aligned}
&\left|\operatorname{proj}_{\theta}\left(\bigcup_{k \in \mathscr{G}_{\mu}^{\theta}} R_{k}^{(n)}\right)\right| \leq\left|\operatorname{proj}_{\theta}\left(\bigcup_{k \in \mathscr{G}_{\mu}^{\theta}} J_{k}^{(n)}\right)\right| \\
&+\operatorname{Const}\left(\frac{\pi}{2}-|\theta|\right)^{-1} \max _{k \in \mathscr{S}_{\mu}^{\theta}}\left|\operatorname{proj}_{\theta}\left(J_{k}^{(n)}\right)\right| \\
& \leq \text { Const }\left(\frac{\pi}{2}-|\theta|\right)^{-1}\left|\operatorname{proj}_{\theta}\left(\bigcup_{k \in \mathscr{G}_{\mu}^{\theta}} J_{k}^{(n)}\right)\right| \quad\left(1 \leq \mu \leq \nu_{\theta}\right),
\end{aligned}
$$


and hence

$$
\begin{aligned}
\left|\operatorname{proj}_{\theta}(\Gamma)\right| & \leq \text { Const }\left(\frac{\pi}{2}-|\theta|\right)^{-1} \sum_{\mu=1}^{\nu_{\theta}}\left|\operatorname{proj}_{\theta}\left(\bigcup_{k \in \mathscr{G}_{\mu}^{\theta}} J_{k}^{(n)}\right)\right| \\
& =\text { Const }\left(\frac{\pi}{2}-|\theta|\right)^{-1}\left|\operatorname{proj}_{\theta}\left(\Gamma_{n}\right)\right| .
\end{aligned}
$$

We have, for any $0<\varepsilon<\pi / 2$,

$$
\begin{aligned}
\int_{-(\pi / 2)+\varepsilon}^{(\pi / 2)-\varepsilon}\left|\operatorname{proj}_{\theta}(\Gamma)\right| d \theta & \leq \text { Const } \int_{-(\pi / 2)+\varepsilon}^{(\pi / 2)-\varepsilon}\left(\frac{\pi}{2}-|\theta|\right)^{-1}\left|\operatorname{proj}_{\theta}\left(\Gamma_{n}\right)\right| d \theta \\
& \leq \text { Const } \varepsilon^{-1} B u\left(\Gamma_{n}\right) .
\end{aligned}
$$

Since $\liminf _{n \rightarrow \infty} B u\left(\Gamma_{n}\right)=0$, this shows that the first quantity equals zero. Since $0<\varepsilon<\pi / 2$ is arbitrary, $B u(\Gamma)=0$. This completes the proof of Lemma 4.

4. Construction of $E_{0}$. Let $p_{n}$ be the integral part of $(3 / 2)^{4 n / 3}$ $(n \geq 1)$. We define a sequence $\{n(k)\}_{k=1}^{\infty}$ of positive integers by $n(1)=10$,

$$
n(k+1)=10 n(k)+p_{10 n(k)} \quad(k \geq 1) .
$$

We define a sequence $\left\{\varphi_{j}\right\}_{j=0}^{\infty}$ of non-negative numbers by $\varphi_{0}=0$,

$$
\begin{array}{ll}
\varphi_{j}=\frac{1}{2} & (1 \leq j \leq n(1)), \\
\varphi_{j}=\frac{1}{2} & (n(k)<j \leq 10 n(k), k \geq 1), \\
\varphi_{j}=0 & (10 n(k)<j \leq n(k+1), k \geq 1) .
\end{array}
$$

We use Lemma 2 with $\Gamma_{0}, g_{0}=1$ and $\left\{\varphi_{j}\right\}_{j=0}^{10 n(1)}$. There exist a crank $\Gamma_{10 n(1)}$ of type $\left\{\varphi_{j}\right\}_{j=0}^{10 n(1)}$ and a non-negative function $g_{10 n(1)}$ on $\Gamma_{10 n(1)}$ such that $g_{10 n(1)}$ is a constant on each component of $\Gamma_{10 n(1)}$,

$$
\left\|g_{10 n(1)}\right\|_{L^{1}\left(\Gamma_{10 n(1)}\right)}=1, \quad\left\|g_{10 n(1)}\right\|_{L^{\infty}\left(\Gamma_{10 n(1)}\right)} \leq 1 / \prod_{\mu=1}^{10 n(1)}\left(1+\varphi_{\mu}\right)
$$

$\left\|\operatorname{Re} \mathscr{H}_{\Gamma_{10 n(1)}} g_{10 n(1)}\right\|_{L^{\infty}\left(\Gamma_{10 n(1)}\right)}$

$$
\begin{aligned}
& \leq\left\|\operatorname{Re} \mathscr{H}_{\Gamma_{0}} g_{0}\right\|_{L^{\infty}\left(\Gamma_{0}\right)}+\sum_{j=1}^{10 n(1)} 1 / \prod_{\mu=1}^{j}\left(1+\varphi_{\mu}\right) \\
& =\left\{\sum_{j=1}^{n(1)} 1 / \prod_{\mu=1}^{j}\left(1+\varphi_{\mu}\right)\right\}+\sum_{j=n(1)+1}^{10 n(1)} 1 / \prod_{\mu=1}^{j}\left(1+\varphi_{\mu}\right) .
\end{aligned}
$$


Using Lemma 3 with $n=10 n(1), m=p_{10 n(1)}$, we obtain a crank $\Gamma_{n(2)}$ of type $\left\{\varphi_{j}\right\}_{j=0}^{n(2)}$ and a non-negative function $g_{n(2)}$ on $\Gamma_{n(2)}$ such that $g_{n(2)}$ is a constant on each component of $\Gamma_{n(2)}$,

$$
\begin{gathered}
\left\|g_{n(2)}\right\|_{L^{1}\left(\Gamma_{n(2)}\right)}=\left\|g_{10 n(1)}\right\|_{L^{1}\left(\Gamma_{10 n(1)}\right)}=1, \\
\left\|g_{n(2)}\right\|_{L^{\infty}\left(\Gamma_{n(2)}\right)} \leq C_{0}\left\|g_{10 n(1)}\right\|_{L^{\infty}\left(\Gamma_{10 n(1)}\right)} \leq C_{0} / \prod_{\mu=1}^{10 n(1)}\left(1+\varphi_{\mu}\right),
\end{gathered}
$$

$$
\begin{aligned}
\left\|\operatorname{Re} \mathscr{H}_{\Gamma_{n(2)}} g_{n(2)}\right\|_{L^{\infty}\left(\Gamma_{n(2)}\right)} & \leq\left\|\operatorname{Re} \mathscr{H}_{\Gamma_{10 n(1)}} g_{10 n(1)}\right\|_{L^{\infty}\left(\Gamma_{10 n(1)}\right)}+C_{0} \sqrt{p_{10 n(1)}}\left\|g_{10 n(1)}\right\|_{L^{\infty}\left(\Gamma_{10 n(1)}\right)} \\
\leq & \left\{\sum_{j=1}^{n(1)} 1 / \prod_{\mu=1}^{j}\left(1+\varphi_{\mu}\right)\right\}+\sum_{j=n(1)+1}^{10 n(1)} 1 / \prod_{\mu=1}^{j}\left(1+\varphi_{\mu}\right) \\
& +C_{0} \sqrt{p_{10 n(1)}} / \prod_{\mu=1}^{10 n(1)}\left(1+\varphi_{\mu}\right),
\end{aligned}
$$

$$
B u\left(\Gamma_{n(2)}\right) \leq C_{0}\left|\Gamma_{10 n(1)}\right| / p_{10 n(1)}^{9 / 10}=C_{0} \prod_{\mu=1}^{10 n(1)}\left(1+\varphi_{\mu}\right) / p_{10 n(1)}^{9 / 10},
$$

where $C_{0}=\max \left\{C_{1}, C_{2}\right\}$. Using Lemma 2 with $n=n(2), m=9 n(2)$, we obtain a crank $\Gamma_{10 n(2)}$ and a non-negative function $g_{10 n(2)}$. Using Lemma 3 with $n=10 n(1), m=p_{10 n(2)}$, we obtain a crank $\Gamma_{n(3)}$ and a non-negative function $g_{n(3)}$. Repeating this argument, we obtain a crank $\Gamma_{n(k)}(k \geq 2)$ of type $\left\{\varphi_{j}\right\}_{j=0}^{n(k)}$ and a non-negative function $g_{n(k)}$ on $\Gamma_{n(k)}$ such that $g_{n(k)}$ is a constant on each component of $\Gamma_{n(k)}$,

$$
\begin{gathered}
\left\|g_{n(k)}\right\|_{L^{1}\left(\Gamma_{n(k)}\right)}=1 \\
\left\|g_{n(k)}\right\|_{L^{\infty}\left(\Gamma_{n(k)}\right)} \leq C_{0}^{k-1} / \prod_{\mu=1}^{10 n(k-1)}\left(1+\varphi_{\mu}\right),
\end{gathered}
$$

$$
\begin{aligned}
& \left\|\operatorname{Re} \mathscr{\mathcal { H }}_{\Gamma_{n(k)}} g_{n(k)}\right\|_{L^{\infty}\left(\Gamma_{n(k)}\right)} \\
& \leq\left\{\sum_{j=1}^{n(1)} 1 / \prod_{\mu=1}^{j}\left(1+\varphi_{\mu}\right)\right\}+\sum_{\nu=1}^{k-1} \sum_{j=n(\nu)+1}^{10 n(\nu)}\left\{C_{0}^{\nu-1} / \prod_{\mu=1}^{j}\left(1+\varphi_{\mu}\right)\right\} \\
& \quad+\sum_{\nu=1}^{k-1}\left\{C_{0}^{\nu} \sqrt{p_{10 n(\nu)}} / \prod_{\mu=1}^{10 n(\nu)}\left(1+\varphi_{\mu}\right)\right\}
\end{aligned}
$$




$$
B u\left(\Gamma_{n(k)}\right) \leq C_{0} \prod_{\mu=1}^{10 n(k-1)}\left(1+\varphi_{\mu}\right) / p_{10 n(k-1)}^{9 / 10} .
$$

Let $\Gamma=\bigcap_{j=1}^{\infty} \overline{\bigcup_{k=2}^{\infty} \Gamma_{n(k)}}$. Then $\Gamma$ is a crank of type $\left\{\varphi_{j}\right\}_{j=0}^{\infty}$. We have

$$
\begin{aligned}
B u\left(\Gamma_{n(k)}\right) & \leq C_{0} \prod_{\mu=1}^{10 n(k-1)}\left(1+\varphi_{\mu}\right) p_{10 n(k-1)}^{-9 / 10} \\
& \leq \text { Const }\left(\frac{3}{2}\right)^{10 n(k-1)}\left(\frac{3}{2}\right)^{-(4 / 3)(9 / 10) 10 n(k-1)} \\
& =\text { Const }\left(\frac{3}{2}\right)^{-2 n(k-1)},
\end{aligned}
$$

which shows that $\lim _{k \rightarrow \infty} B u\left(\Gamma_{n(k)}\right)=0$. Hence Lemma 4 gives that $B u(\Gamma)=0$.

We now show that $\gamma(\Gamma)>0$. Let $k \geq 1$. Then

$$
\int_{\Gamma_{n(k)}} g_{n(k)}(\zeta)|d \zeta|=1
$$

Since $n(\nu) \geq 10 n(\nu-1)(\nu \geq 2), n(1)=10$, we have $n(\nu) \geq 10^{\nu}(\nu \geq$ $1)$, and hence

$$
\left\|g_{n(k)}\right\|_{L^{\infty}\left(\Gamma_{n(k)}\right)} \leq C_{0}^{k-1}\left(\frac{3}{2}\right)^{-9 n(k-1)} \leq \text { Const. }
$$

Since

$$
\begin{gathered}
\sqrt{p_{10 n(\nu)}}\left\{\prod_{\mu=1}^{10 n(\nu)}\left(1+\varphi_{\mu}\right)\right\}^{-1} \leq \sqrt{p_{10 n(\nu)}}\left(\frac{3}{2}\right)^{-9 n(\nu)} \\
\leq \text { Const }\left(\frac{3}{2}\right)^{(4 / 3)(1 / 2) 10 n(\nu)}\left(\frac{3}{2}\right)^{-9 n(\nu)} \\
=\text { Const }\left(\frac{3}{2}\right)^{-(7 / 3) n(\nu)} \quad(\nu \geq 1)
\end{gathered}
$$

we have

$$
\left\|\operatorname{Re} \mathscr{F}_{\Gamma_{n(k)}} g_{n(k)}\right\|_{L^{\infty}\left(\Gamma_{n(k)}\right)} \leq \text { Const. }
$$

Hence we can define a non-negative function $h_{k}$ on $\Gamma_{n(k)}$ so that

$$
\begin{aligned}
& \int_{\Gamma_{n(k)}} h_{k}(\zeta)|d \zeta|=\eta_{0}, \quad\left\|h_{k}\right\|_{L^{\infty}\left(\Gamma_{n(k)}\right)} \leq 1 / 2, \\
& \left\|\operatorname{Re} \mathscr{F}_{\Gamma_{n(k)}}, h_{k}\right\|_{L^{\infty}\left(\Gamma_{n(k)}\right)} \leq 1 / 2, \\
& h_{k}(\zeta)=0 \text { at endpoints of each component of } \Gamma_{n(k)}, \\
& h_{k} \text { is differentiable along } \Gamma_{n(k)},
\end{aligned}
$$


where $\eta_{0}$ is an absolute constant. Let

$$
\begin{gathered}
\hat{h}_{k}(z)=\frac{1}{2 \pi i} \int_{\Gamma_{n(k)}} \frac{h_{k}(\zeta)}{\zeta-z}|d \zeta|, \\
u_{k}(z)=\operatorname{Re} \hat{h}_{k}(z), \quad v_{k}(z)=\left(\text { the imaginary part of } \hat{h}_{k}(z)\right), \\
f_{k}(z)=\left\{1-\exp \left(i \hat{h}_{k}(z)\right)\right\} /\left\{1+\exp \left(i \hat{h}_{k}(z)\right)\right\} \quad\left(z \notin \Gamma_{n(k)}\right)
\end{gathered}
$$

(cf. [1, p. 30]). We see easily that $f_{k}$ is analytic outside $\Gamma_{n(k)}$ and

$$
f_{k}^{\prime}(\infty)=\frac{1}{4 \pi} \int_{\Gamma_{n(k)}} h_{k}(\zeta)|d \zeta|=\eta_{0} / 4 \pi
$$

The non-tangential limit of $\left|u_{k}(z)\right|$ to each point on $\Gamma_{n(k)}$ is dominated by

$$
\left\|h_{k}\right\|_{L^{\infty}\left(\Gamma_{n(k)}\right)}+\left\|\operatorname{Re} \mathscr{K}_{\Gamma_{n(k)}} h_{k}\right\|_{L^{\infty}\left(\Gamma_{n(k)}\right)} \leq 1 .
$$

Since $\left|u_{k}\right|$ is sub-harmonic in $\Gamma_{n(k)}^{c}$ and continuous in $\mathbf{C} \cup\{\infty\}$, we have $\sup _{z \in \Gamma_{n(k)}^{c}}\left|u_{k}(z)\right| \leq 1$. Hence, for any $z \notin \Gamma_{n(k)}$,

$$
\left|f_{k}(z)\right|^{2}=\frac{1+\exp \left(-2 v_{k}(z)\right)-2 \exp \left(-v_{k}(z)\right) \cos \left(u_{k}(z)\right)}{1+\exp \left(-2 v_{k}(z)\right)+2 \exp \left(-v_{k}(z)\right) \cos \left(u_{k}(z)\right)} \leq 1,
$$

which shows that $\left\|f_{k}\right\|_{H^{\infty}\left(\Gamma_{n(k)}^{c}\right)} \leq 1$. Since $k \geq 1$ is arbitrary, using an argument of normal families, we obtain $f \in H^{\infty}\left(\Gamma^{c}\right)$ satisfying $f^{\prime}(\infty)=\eta_{0} / 4 \pi,\|f\|_{H^{\infty}\left(\Gamma^{c}\right)} \leq 1$. This shows that $\gamma(\Gamma) \geq \eta_{0} / 4 \pi$. Normalizing $\Gamma$, we obtain the required set $E_{0}$.

\section{REFERENCES}

[1] J. Garnett, Analytic Capacity and Measure, Lecture Notes in Mathematics, Vol. 297, Springer-Verlag, Berlin/New York, 1972.

[2] L. D. Ivanov, On sets of analytic capacity zero, in Linear and Complex Analysis Problem Book (Edited by V. P. Havin, S. V. Hruščëv and N. K. Nikol'skii), Lecture Notes in Mathematics, Vol. 1043, Springer-Verlag, Berlin/New York, 1984, 498-501.

[3] D. E. Marshall, Removable sets for bounded analytic functions, in Linear and Complex Analysis Problem Book (Edited by V. P. Havin, S. V. Hruščëv and N. K. Nikol'skii), Lecture Notes in Mathematics, Vol. 1043, Springer-Verlag, Berlin/New York, 1984, 485-490.

[4] P. Mattila, Smooth maps, null-sets for integral geometric measure and analytic capacity, Annales of Math., 123 (1986), 303-309.

[5] T. Murai, Comparison between analytic capacity and the Buffon needle probability, Trans. Amer. Math. Soc. 304 (1987), to appear.

[6] L. A. Santaló, Introduction to Integral Geometry, Hermann, 1953.

[7] A. G. Vitushkin, Analytic capacity of sets and problems in approximation theory, Russian Math. Surveys, 22 (1967), 139-200.

Received March 27, 1987.

YALE UNIVERSITY

New Haven, CT 06520 


\section{PACIFIC JOURNAL OF MATHEMATICS}

\section{EDITORS}

V. S. VARADARAJAN

(Managing Editor)

University of California

Los Angeles, CA 90024

Herbert Clemens

University of Utah

Salt Lake City, UT 84112

R. FINN

Stanford University

Stanford, CA 94305
HERMANN FLASCHKA

University of Arizona

Tucson, AZ 85721

RAMESh A. GANGOLLI

University of Washington Seattle, WA 98195

VAUGHAN F. R. JONES

University of California

Berkeley, CA 94720
ROBION KIRBY

University of California

Berkeley, CA 94720

C. C. MOORE

University of California

Berkeley, CA 94720

HAROLD STARK

University of California, San Diego

La Jolla, CA 92093

\section{ASSOCIATE EDITORS}
R. AREnS
E. F. BECKENBACH
B. H. NEUMANN
F. WOLF
K. YOSHIDA (1906-1982)

\section{SUPPORTING INSTITUTIONS}

UNIVERSITY OF ARIZONA

UNIVERSITY OF OREGON

UNIVERSITY OF BRITISH COLUMBIA UNIVERSITY OF SOUTHERN CALIFORNIA

CALIFORNIA INSTITUTE OF TECHNOLOGY

UNIVERSITY OF CALIFORNIA

STANFORD UNIVERSITY

MONTANA STATE UNIVERSITY

UNIVERSITY OF HAWAII

UNIVERSITY OF NEVADA, RENO

UNIVERSITY OF TOKYO

NEW MEXICO STATE UNIVERSITY

UNIVERSITY OF UTAH

OREGON STATE UNIVERSITY

WASHINGTON STATE UNIVERSITY

UNIVERSITY OF WASHINGTON 


\section{Pacific Journal of Mathematics}

\section{Vol. 133, No. $1 \quad$ March, 1988}

John Anderson, Finitely generated algebras and algebras of solutions to partial differential equations $\ldots \ldots \ldots \ldots \ldots \ldots \ldots \ldots \ldots \ldots \ldots \ldots \ldots$

Junichi Aramaki, On an extension of the Ikehara Tauberian theorem . . . . . 13

Giacomo Monti Bragadin, Abstract Riemannian stratifications .......... 31

Lawrence James Brenton and Richard Hill, On the Diophantine equation

$1=\sum 1 / n_{i}+1 / \prod n_{i}$ and a class of homologically trivial complex

surface singularities .................................41

C. Bruce Hughes, Controlled homotopy topological structures $\ldots . \ldots \ldots \ldots 69$

Peter Wilcox Jones and Takafumi Murai, Positive analytic capacity but zero Buffon needle probability . ...........................999

Gary M. Lieberman, Hölder continuity of the gradient at a corner for the

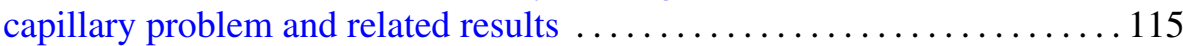

Feng Luo, Representing homology classes of $C \mathbf{P}^{2} \overline{C \mathbf{P}^{2}}$

Claudio Nebbia, Groups of isometries of a tree and the Kunze-Stein

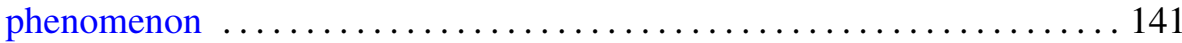

Stefan Richter, Unitary equivalence of invariant subspaces of Bergman and

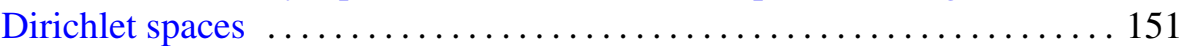

Paul Frederick Ringseth, The Selberg trace formula for groups without Eisenstein series ..................................... 157

Abderrazzak Sersouri, The Mazur property for compact sets $\ldots \ldots \ldots \ldots 185$ Alladi Sitaram, On an analogue of the Wiener Tauberian theorem for symmetric spaces of the noncompact type 